\title{
COMMON PARTIAL TRANSVERSALS AND INTEGRAL MATRICES
}

\author{
BY \\ R. A. BRUALDI $\left({ }^{1}\right)$
}

\begin{abstract}
Certain packing and covering problems associated with the common partial transversals of two families $\mathscr{U}$ and $\mathfrak{B}$ of subsets of a set $E$ are investigated. Under suitable finitary restrictions, necessary and sufficient conditions are obtained for there to exist pairwise disjoint sets $F_{1}, \ldots, F_{t}$ where each $F_{i}$ is a partial transversal of $\mathfrak{A}$ with defect at most $p$ and a partial transversal of $\mathfrak{B}$ with defect at most $q$. We also prove that (i) $E=\bigcup_{i=1}^{t} T_{i}$ where each $T_{i}$ is a common partial transversal of $\mathfrak{U}$ and $\mathfrak{B}$ if and only if (ii) $E=\bigcup_{i=1}^{t} T_{i}^{\prime}$ where each $T_{i}^{\prime}$ is a partial transversal of $\mathfrak{A}$ and (iii) $E=\bigcup_{i=1}^{t} T_{i}^{\prime \prime}$ where each $T_{i}^{\prime \prime}$ is a partial transversal of $\mathfrak{B}$. We then derive necessary and sufficient conditions for the validity of (i).

The proofs are accomplished by establishing a connection with these common partial transversal problems and representations of integral matrices (not necessarily finite or countably infinite) as sums of subpermutation matrices and then using known results about the existence of a single common partial transversal of two families. Accordingly various representation theorems for integral matrices are derived.
\end{abstract}

1. Introduction. Let us begin by describing two general combinatorial problems. We take $E$ to be an arbitrary set, possibly infinite, and $\mathscr{F}$ to be a distinguished collection of subsets of $E$. Let $t$ be a natural number. The packing problem of order $t$ associated with $\mathscr{F}$ is the following: When does there exist a family $\left(F_{i}: 1 \leqq i \leqq t\right)$ of pairwise disjoint sets with $F_{i} \in \mathscr{F}(1 \leqq i \leqq t)$ ? The covering problem of order $t$ associated with $\mathscr{F}$ is: When does there exist a family $\left(F_{i}^{\prime}: 1 \leqq i \leqq t\right)$ of sets with $F_{i}^{\prime} \in \mathscr{F}(1 \leqq i \leqq t)$ such that $E=F_{1}^{\prime} \cup \cdots \cup F_{t}^{\prime}$ ? In a typical packing problem the sets in $\mathscr{F}$ are given a descriptive definition, and it is not assumed that $\mathscr{F}$ is nonvacuous. The packing problem of order 1 is that of the existence of an object in $\mathscr{F}$; the covering problem of order 1 seeks to determine when $E$ is an object in $\mathscr{F}$.

Suppose $\mathfrak{A}=\left(A_{i}: i \in I\right)$ is a family of subsets of the set $E$. Let $\mathscr{F}$ be the collection of transversals of $\mathfrak{A}$. If $I$ is a finite set (i.e. $\mathfrak{A}$ is a finite family), the solution of the packing problem of order $t$ is a special case of a theorem of Higgins [11]; if $I$ is an infinite set but $A_{i}$ is a finite set $(i \in I)$, the solution is a special case of Mirsky's generalization [14] of Higgins' theorem. The covering problem of order $t$ in this

Received by the editors March 10, 1970.

AMS 1969 subject classifications. Primary 0504, 0525, 1548; Secondary 1565, 0460.

Key words and phrases. Packing problem, covering problem, partial transversal, transversal, defect of a partial transversal, common partial transversal, integral matrix, subpermutation matrix, 'row and column defect of a subpermutation matrix, permutation matrix, representations of an integral matrix, linking principle.

( $\left.{ }^{1}\right)$ Research supported by a NATO Postdoctoral Fellowship at the University of Sheffield. Copyright (C) 1971, American Mathematical Society 
case, with suitable finiteness restrictions, has also been solved. This will turn out to be a special case of a more general covering problem we shall solve here.

A general class of packing and covering problems has been solved by Edmonds [4]. Let $E$ be a finite set and $\mathscr{E}$ an independence structure or matroid on $E$. Thus $\mathscr{E}$ is a nonvacuous collection of subsets of $E$ satisfying

(i) $A \in \mathscr{E}, A^{\prime} \subseteq A$ imply $A^{\prime} \in \mathscr{E}$.

(ii) $A, B \in \mathscr{E},|A|+1=|B|$ imply there is a $b \in B \backslash A$ such that $A \cup\{b\} \in \mathscr{E}$.

The axioms for an independence structure are taken from properties of linear independence of vectors in a vector space. Let $\mathscr{F}$ be the collection of bases of $\mathscr{E}$, that is members of $\mathscr{E}$ which are maximal with respect to set-theoretic inclusion. Both the packing and covering problems of order $t$ for $\mathscr{F}$ have been solved by Edmonds [4], the results being further extended by Edmonds and Fulkerson [5]. The finite packing and covering problems of the preceding paragraph are special cases of this one.

It is true that most packing and covering problems for which a solution is at hand fall into the category of the preceding paragraph. Recently, Fulkerson [8] has solved a packing problem which does not fall into this category. Let $\mathfrak{A}=$ $\left(A_{i}: i \in I\right)$ and $\mathfrak{B}=\left(B_{j}: j \in J\right)$ be two finite families of subsets of a finite set $E$. Let $\mathscr{F}$ be the collection of common transversals of $\mathfrak{A}$ and $\mathfrak{B}$. Now it is well known that $\mathscr{F}$ is not in general the collection of bases of a matroid on $E$. Nevertheless Fulkerson, using the theory of flows in networks, solved the packing problem of order $t$ associated with $\mathscr{F}\left({ }^{2}\right)$ and, more generally, that associated with $\mathscr{F}_{p}$, the collection of common partial transversals of size $p$ of $\mathfrak{A}$ and $\mathfrak{B}$.

Generalizing Fulkerson's theorem and the broad structure of his proof (our work, however, was done independently), we shall solve here the packing problem of order $t$ associated with the common transversals of two arbitrary families $\mathfrak{A}$ and $\mathfrak{B}$, of subsets of a set $E$ (with suitable finitary restrictions on the subsets) and, more generally, that associated with the common partial transversals with defect at most $d$. We shall also solve the covering problem of order $t$ associated with the common partial transversals of $\mathfrak{A}$ and $\mathfrak{B}$. Surprisingly, it turns out that this covering problem is equivalent to the covering problem of order $t$ associated with the partial transversals of $\mathfrak{A}$ and that associated with the partial transversals of $\mathfrak{B}$. Our solutions depend on known criteria for the existence of transversals and common transversals (this is the essential difference with Fulkerson's approach and it has a simplifying influence) and some representation theorems for integral matrices. Accordingly, after a brief review of some results in transversal theory, we shall be concerned with generalizing some known representation theorems for finite integral matrices to arbitrary integral matrices. The proofs of these results, of interest on their own, will be based on an existence theorem for partial transversals with additional properties.

(2) In a paper to be published, J. de Sousa also gives a solution to this problem. 
For sets $X$ and $Y$, we shall use $|X|$ to denote the cardinal number of $X$, which is either a nonnegative integer or $\infty$, and $X \backslash Y$ to denote the set-theoretic difference of $X$ and $Y$. We shall also use Rado's notation $Y \subset \subset X$ to mean $Y$ is a finite subset of $X$. Also, if $\mathfrak{A}(I)=\left(A_{i}: i \in I\right)$ is a family of sets and $K \subseteq I, \mathfrak{A}(K)=\left(A_{i}: i \in K\right)$ is a subfamily of $\mathfrak{Q}(I)$ and $A(K)=\bigcup_{i \in K} A_{i}$.

2. Some known results in transversal theory. Let $\mathfrak{A}(I)=\left(A_{i}: i \in I\right)$ be a family, indexed by a set $I$, of subsets of $E$. For short, we shall denote, sometimes, this family by $\mathfrak{A}$. A set $T \subseteq E$ is a transversal of $\mathfrak{A}$ provided there exists a bijection $\theta: T \rightarrow I$ such that

$$
x \in A_{\theta(x)} \quad(x \in T) .
$$

If $\theta$ is only an injection satisfying (2.1), then $T$ is a partial transversal of $\mathfrak{A}$, and the defect of $T$ in $\mathfrak{A}$ is defined by

$$
d_{\mathfrak{U}}(T)=\min \{|I| \theta(T) \mid: \theta: T \rightarrow I, \text { an injection satisfying (2.1)\}. }
$$

Thus a transversal of $\mathfrak{A}$ is a partial transversal with defect equal to 0 . The definition of defect given by (2.2) can be simplified if $I$ is a finite set; it is simply $|I \backslash \theta(T)|$ where $\theta: T \rightarrow I$ is any injection satisfying (2.1). If $I$ is an infinite set, $|I \backslash \theta(T)|$ may vary with the injection $\theta$. For instance, if $I=E$ is the set of positive integers and $A_{i}=\{i, i+1\}(i \in I)$, then $T=I \backslash\{1\}$ is a partial transversal of $\mathfrak{A}$. The injection $\theta_{1}: T \rightarrow I$ defined by $\theta_{1}(i)=i-1(i \in I)$ satisfies (2.1) and $\left|\theta_{1}(T) \backslash I\right|=0$; the injection $\theta_{2}: T \rightarrow I$ defined by $\theta_{2}(i)=i(i \in I)$ satisfies $(2.1)$ and $\left|\theta_{2}(T)\right| I \mid=1$.

If $\mathfrak{A}(I)=\left(A_{i}: i \in I\right)$ is a family of finite subsets of a set $E$, then necessary and sufficient conditions for $\mathfrak{A}$ to have a transversal are $|A(K)| \geqq|K|(K \subset \subset I)$. If $I$ is a finite set, in which case it is not necessary to assume the $A$ 's are finite sets, this is P. Hall's theorem [9]; if $I$ is infinite, this is M. Hall, Jr.'s theorem [10]. In a previous paper we proved a quite general theorem (Theorem 3 of [1]; for a related result see [6]) which contains these as well as most other theorems on the existence of partial transversals as special cases. We will not use this theorem, which is a transfinite and symmetrical version of a result of Hoffman and Kuhn [12], in its full generality, and therefore we will be content to extract a weakened version sufficient to fulfill our requirements.

If $\mathfrak{X}(I)=\left(A_{i}: i \in I\right)$ is a family of subsets of a set $E$, then the dual family $\mathfrak{A}^{*}(E)=\left(A_{e}^{*}: e \in E\right)$ is defined by

$$
A_{e}^{*}=\left\{i \in I: e \in A_{i}\right\} \quad(e \in E) .
$$

THEOREM 2.1. Let $\mathfrak{A}(I)=\left(A_{i}: i \in I\right)$ be a family of finite subsets of a set $E$ such that each element of $E$ is a member of only finitely many $A$ 's (i.e. the sets in the dual family are finite). Let the sets $I_{1}, I_{2}$ partition $I$ and the sets $E_{1}, E_{2}$ partition $E$. Let there be given integers $c_{k}, c_{k}^{\prime}$ with $0 \leqq c_{k} \leqq c_{k}^{\prime}(k=1,2)$ and integers $d_{l}, d_{l}^{\prime}$ with $0 \leqq d_{l} \leqq d_{l}^{\prime}(l=1,2)$. If $c_{k}>0$, assume $I_{k}$ is a finite set $(k=1,2)$; if $d_{l}>0$, assume $E_{l}$ is a finite set $(l=1,2)$. 
Then there exists $\bar{I} \subseteq I, \bar{E} \subseteq E$ such that $\bar{E}$ is a transversal of $\mathfrak{A}(\bar{I})$ with $c_{k} \leqq\left|I_{k}\right| \bar{I} \mid$ $\leqq c_{k}^{\prime}(k=1,2), d_{l} \leqq\left|E_{l}\right| \bar{E} \mid \leqq d_{l}^{\prime}(l=1,2)$ if and only if the following conditions are satisfied.
(i) $|A(U)|+\sum_{\left\{k: U \cap I_{k} \neq \varnothing\right\}} c_{k}^{\prime} \geqq|U|(U \subset \subset I)$.
(ii) $\left|A(U) \cap E_{1}\right|+\sum_{\left\{k: U \cap I_{k} \neq \varnothing\right)} c_{k}^{\prime}+\left|E_{2}\right|-d_{2} \geqq|U|(U \subset \subset I)$.
(iii) $\left|A(U) \cap E_{2}\right|+\sum_{\left\{k: U \cap I_{k} \neq \varnothing\right\}} c_{k}^{\prime}+\left|E_{1}\right|-d_{1} \geqq|U|(U \subset \subset I)$.

(2.4) The dual inequalities.

It is permissible that $c_{k}^{\prime}=\infty(k=1,2)$ and $d_{l}^{\prime}=\infty(l=1,2)$. If one takes $d_{l}^{\prime}=\infty$ $(l=1,2)$ and $c_{k}=0(k=1,2)$, then we conclude that the conditions of (2.3) are necessary and sufficient for there to exist $\bar{I} \subseteq I, \bar{E} \subseteq E$ such that $\bar{E}$ is a transversal of $\mathfrak{A}(\bar{I})$ where $\left|I_{k}\right| \bar{I} \mid \leqq c_{k}^{\prime}(k=1,2)$ and $d_{l} \leqq\left|E_{l}\right| \bar{E} \mid(l=1,2)$. An analogous conclusion can be arrived at by taking $c_{k}^{\prime}=\infty(k=1,2)$ and $d_{l}=0(l=1,2)$.

Now suppose $\mathfrak{A}(I)=\left(A_{i}: i \in I\right)$ and $\mathfrak{B}(J)=\left(B_{j}: j \in J\right)$ are two families of subsets of a set $E$. A set $T \subseteq E$ is a common partial transversal of $\mathfrak{A}$ and $\mathfrak{B}$ provided it is a partial transversal of both. A common transversal is defined analogously. In [2] the principal result of [1] was generalized to common partial transversals. We shall not need this theorem in its full generality, so again we will be content to record a version sufficient for our purposes.

TheOREM 2.2. Let $\mathfrak{A}(I)=\left(A_{i}: i \in I\right)$ and $\mathfrak{B}(J)=\left(B_{j}: j \in J\right)$ be two families of subsets of a set $E$. Assume that each element of $E$ is a member of only finitely many $A$ 's and B's. Let $p$ and $q$ be nonnegative integers or $\infty$. If $p<\infty$, assume $A_{i}$ is a finite set $(i \in I)$; if $q<\infty$, assume $B_{j}$ is a finite set $(j \in J)$. Let $M \subseteq E$. Then there exists a set $T \subseteq E$ with $M \subseteq T$ such that $T$ is a common partial transversal of $\mathfrak{A}$ and $\mathfrak{B}$ with $d_{\mathfrak{U}}(T) \leqq p, d_{\mathfrak{B}}(T) \leqq q$ if and only if

$$
\begin{aligned}
& |A(P) \cap B(J \backslash Q)|+|\{A(P) \cup B(J \backslash Q)\} \cap N|+p \geqq|P|-|Q|+|N| \\
& (P \subset \subset I, Q \subset \subset J, N \subset \subset M), \\
& |A(I \backslash P) \cap B(Q)|+|\{A(I \backslash P) \cup B(Q)\} \cap N|+q \geqq|Q|-|P|+|N| \\
& (P \subset \subset I, Q \subset \subset J, N \subset \subset M) \text {, } \\
& |A(I \backslash P) \cap N|+|P| \geqq|N| \quad(P \subset \subset I, N \subset \subset M), \\
& |B(J \backslash Q) \cap N|+|Q| \geqq|N| \quad(Q \subset \subset J, N \subset \subset M) .
\end{aligned}
$$

Observe that if we take $M=\varnothing,(2.5)$ simplifies to

$$
|A(P) \cap B(J \backslash Q)|+p \geqq|P|-|Q| \quad(P \subset \subset I, Q \subset \subset J) .
$$

A similar simplification occurs for (2.6) while (2.7) and (2.8) disappear. If $|I|=|J|=n<\infty$, then we obtain a result of Ford and Fulkerson [7]. Also note that if $p=q=\infty,(2.5)$ and (2.6) 'disappear' so that (2.7) and (2.8) are necessary and sufficient for $M$ to be a common partial transversal of $\mathfrak{A}$ and $\mathfrak{B}$. 
From Theorem 2.2 a theorem about a single family of sets can be obtained.

COROLlary 1. Let $\mathfrak{U}(I)=\left(A_{i}: i \in I\right)$ be a family of subsets of $E$ such that each element of $E$ is a member of only finitely many A's. Let $p$ be a nonnegative integer or $\infty$, and assume that $A_{i}$ is a finite set $(i \in I)$ if $p<\infty$. Then $E$ is a transversal of $\mathfrak{A}$ with $d_{\mathfrak{A}}(E) \leqq p$ if and only if

$$
\begin{array}{rlrl}
|A(P)|+p & \geqq|P| & & (P \subset \subset I), \\
|A(I \mid P) \cap F|+|P| & \geqq|F| & (P \subset \subset I, F \subset \subset E) .
\end{array}
$$

This corollary can be obtained from Theorem 2.2 by choosing $M=\varnothing, \mathfrak{B}=$ $\left(B_{e}: e \in E\right)$ where $B_{e}=\{e\}(e \in E)$ and $q=0$.

3. Integral matrices. We shall take a rather broad view of the notion of a matrix. It will be simply a family of elements indexed by the cartesian product of two sets. Thus $C=\left(c_{i j}: i \in I, j \in J\right)$ denotes a matrix indexed by the cartesian product of $I$ and $J$, sometimes referred to as an $(I, J)$-matrix. The sets $I$ and $J$ need not be finite nor countably infinite. The notions of row and column still make good sense, $I$ indexes the rows while $J$ indexes the columns. For instance, the family $\left(c_{i j}: j \in J\right)$ is the $i$ th row $(i \in I)$. One can add two $(I, J)$-matrices by adding corresponding entries. We shall only be considering nonnegative integral matrices, matrices all of whose entries are nonnegative integers.

Let $\left(a_{k}: k \in K\right)$ be a family of nonnegative integers indexed by $K$. If $K^{*}=$ $\left\{k \in K: a_{k}>0\right\}$, then $\sum_{k \in K} a_{k}=\infty$ if $K^{*}$ is an infinite set while $\sum_{k \in K} a_{k}=\sum_{k \in K^{*}} a_{k}$ if $K^{*}$ is a finite set. The $i$ th row sum of the matrix $C$ defined above is $\sum_{j \in J} c_{i j}(i \in I)$, while the $j$ th column sum is $\sum_{i \in I} c_{i j}(j \in J)$.

A matrix $P=\left(p_{i j}: i \in I, j \in J\right)$ is a subpermutation matrix if $p_{i j}=0$ or $1(i \in I$, $j \in J$ ) with at most one 1 in each row and column. Thus if $P$ is a subpermutation matrix, all of its row and column sums are either 0 or 1 . If $I^{\prime}=\left\{i \in I: \sum_{j \in J} p_{i j}=1\right\}$ and $J^{\prime}=\left\{j \in J: \sum_{i \in I} p_{i j}=1\right\}$, then $P$ has row defect $\left|I \backslash I^{\prime}\right|$ and column defect $\left|J \backslash J^{\prime}\right|$. The defects are either nonnegative integers or $\infty$. If $I^{\prime}$ is a finite set, then so is $J^{\prime}$ and $\left|I^{\prime}\right|=\left|J^{\prime}\right|$ and we say that $P$ has rank $\left|I^{\prime}\right|$. If the row defect (resp. column defect) of $P$ is 0 , then $P$ is called a row permutation matrix (resp. column permutation matrix). If both the row and column defect are 0 , then $P$ is a permutation matrix. Thus a permutation matrix has exactly one 1 in each row and column.

If $C=\left(c_{i j}: i \in I, j \in J\right)$ and $D=\left(d_{i j}: i \in I, j \in J\right)$ are integral matrices, then we write as usual $C \leqq D$ to mean $c_{i j} \leqq d_{i j}(i \in I, j \in J)$. We are now prepared to prove the first theorem about integral matrices from which most of our other results on integral matrices will follow.

THEOREM 3.1. Let $C=\left(c_{i j}: i \in I, j \in J\right)$ be a nonnegative integral matrix with

$$
\text { (i) } \sum_{j \in J} c_{i j} \leqq t(i \in I), \quad \text { (ii) } \quad \sum_{i \in I} c_{i j} \leqq t \quad(j \in J)
$$


where $t$ is a positive integer. Let

$$
\begin{aligned}
& t(p-1)+1 \leqq \sum_{i \in I}\left(t-\sum_{j \in J} c_{i j}\right) \leqq t p^{\prime} \\
& t(q-1)+1 \leqq \sum_{j \in J}\left(t-\sum_{i \in I} c_{i j}\right) \leqq t q^{\prime}
\end{aligned}
$$

where $p, q$ are nonnegative integers, $p^{\prime}, q^{\prime}$ are nonnegative integers or $\infty$ with $p \leqq p^{\prime}$, $q \leqq q^{\prime}$ where if $p>0$ (resp. $\left.q>0\right)$, then $p^{\prime}<\infty$ (resp. $\left.q^{\prime}<\infty\right)$. Then there exists $a$ subpermutation matrix $P$ with the properties

(3.4) $P \leqq C$,

(3.5) $P$ has $a 1$ in each row and column of $C$ which sums to $t$,

(3.6) $p \leqq($ row defect of $P) \leqq p^{\prime}, q \leqq($ column defect of $P) \leqq q^{\prime}$.

Proof. Before beginning the proof we remark that (3.2) and (3.3) are not conditions restricting the matrices to which the theorem may be applied. Given $C$ satisfying (3.1) we determine $p, p^{\prime}, q, q^{\prime}$ so that (3.2) and (3.3) are satisfied.

We define a family $\mathfrak{A}(I)=\left(A_{i}: i \in I\right)$ of subsets of $J$ by

$$
A_{i}=\left\{j \in J: c_{i j} \neq 0\right\} \quad(i \in I) .
$$

Let $I_{1}=\left\{i \in I: \sum_{j \in J} c_{i j}=t\right\}$ and $I_{2}=I \backslash I_{1}$. Likewise, let $J_{1}=\left\{j \in J: \sum_{i \in I} c_{i j}=t\right\}$ and $J_{2}=J \backslash J_{1}$. The existence of a subpermutation matrix $P$ satisfying (3.4), (3.5) and (3.6) is equivalent to the existence of $\bar{I} \subseteq I, \bar{J} \subseteq J$ such that $\bar{J}$ is a transversal of $\mathfrak{X}(\bar{I})$ where

$$
\begin{array}{ll}
0 \leqq\left|I_{1}\right| \bar{I} \mid \leqq 0, & p \leqq\left|I_{2}\right| \bar{I} \mid \leqq p^{\prime}, \\
0 \leqq\left|J_{1}\right| \bar{J} \mid \leqq 0, & q \leqq\left|J_{2}\right| \bar{J} \mid \leqq q^{\prime}
\end{array}
$$

Because of condition (3.1), both the family $\mathfrak{A}(I)$ and its dual family are families of finite sets. Moreover, if $p>0$ (resp. $q>0$ ), then since $p^{\prime}<\infty$ (resp. $\left.q^{\prime}<\infty\right), I_{2}$ (resp. $J_{2}$ ) is a finite set. Thus we are able to apply Theorem 2.1 and we need only verify that conditions (2.3) and (2.4) hold where $c_{1}=0, c_{2}=p, c_{1}^{\prime}=0, c_{2}^{\prime}=p^{\prime}, d_{1}=0$, $d_{2}=q, d_{1}^{\prime}=0, d_{2}^{\prime}=q^{\prime}$. Because of the symmetry present we need only concern ourselves with conditions (2.3). Each of the three parts of (2.3) divide conveniently into two. Thus there are altogether six conditions to be verified. We state and prove them in order. Whenever $U \subseteq I$, let $U_{1}=U \cap I_{1}$ and $U_{2}=U \cap I_{2}$.

$$
|A(U)| \geqq|U| \quad\left(U \subset \subset I_{1}\right) .
$$

Suppose for some $U \subset \subset I_{1},|A(U)|<|U|$. Then by (3.1)(ii),

$$
\sum_{i \in U} \sum_{j \in J} c_{i j}<t|U|
$$

which contradicts the fact that $\sum_{j \in J} c_{i j}=t\left(i \in I_{1}\right)$.

$$
|A(U)|+p^{\prime} \geqq|U| \quad\left(U \subset \subset I, U_{2} \neq \varnothing\right) .
$$


Suppose for some $U \subset \subset I,|A(U)|+p^{\prime}<|U|$. Then by (3.1)(ii),

$$
\sum_{i \in U_{1}} \sum_{j \in J} c_{i j}+\sum_{i \in U_{2}} \sum_{j \in J} c_{i j}<t\left|U_{1}\right|+t\left(\left|U_{2}\right|-p^{\prime}\right),
$$

or, since $\sum_{j \in J} c_{i j}=t\left(i \in U_{1}\right)$,

$$
\sum_{i \in U_{2}} \sum_{j \in J} c_{i j}<t\left|U_{2}\right|-t p^{\prime}
$$

and thus

$$
t p^{\prime}<t\left|U_{2}\right|-\sum_{i \in U_{2}} \sum_{j \in J} c_{i j}
$$

This contradicts (3.2).

$$
\left|A(U) \cap J_{1}\right|+\left|J_{2}\right|-q \geqq|U| \quad\left(U \subset \subset I_{1}\right) .
$$

Suppose for some $U \subset \subset I_{1},\left|A(U) \cap J_{1}\right| \leqq|U|+q-\left|J_{2}\right|-1$. Then

$$
\sum_{i \in U} \sum_{j \in J_{1}} c_{i j} \leqq t\left(|U|+q-\left|J_{2}\right|-1\right) .
$$

Since $U \subseteq I_{1}$, this implies

$$
t\left|J_{2}\right|-\sum_{j \in J_{2}} \sum_{i \in U} c_{i j} \leqq t(q-1),
$$

and this contradicts (3.3).

$$
\left|A(U) \cap J_{1}\right|+p^{\prime}+\left|J_{2}\right|-q \geqq|U| \quad\left(U \subset \subset I, U_{2} \neq \varnothing\right) .
$$

Suppose for some $U \subset \subset I$, this inequality did not hold. Then

$$
\begin{aligned}
\sum_{i \in U} \sum_{j \in J_{1}} c_{i j} & \leqq t\left(|U|+q-p^{\prime}-\left|J_{2}\right|-1\right), \\
t\left(p^{\prime}+\left|J_{2}\right|+1-q\right) & \leqq t|U|-\sum_{i \in U} \sum_{j \in J_{1}} c_{i j} \\
& =\sum_{i \in U} \sum_{j \in J_{2}} c_{i j}+t\left|U_{2}\right|-\sum_{i \in U_{2}} \sum_{j \in J} c_{i j} .
\end{aligned}
$$

Since $t\left|J_{2}\right|-\sum_{j \in J_{2}} \sum_{i \in U} c_{i j} \geqq t(q-1)+1$ by (3.3), this means that $t p^{\prime}+1 \leqq t\left|U_{2}\right|$ $-\sum_{i \in U_{2}} \sum_{j \in J} c_{i j}$, and this contradicts (3.2).

$$
\left|A(U) \cap J_{2}\right|+\left|J_{1}\right| \geqq U \quad\left(U \subset \subset I_{1}\right) .
$$

If for some $U \subset \subset I_{1}$ the inequality does not hold, then

$$
\begin{aligned}
\sum_{i \in U} \sum_{j \in J_{2}} c_{i j} & <t\left(|U|-\left|J_{1}\right|\right), \\
t\left|J_{1}\right| & <t|U|-\sum_{i \in U} \sum_{j \in J_{2}} c_{i j}, \\
t\left|J_{1}\right| & <\sum_{i \in U} \sum_{j \in J_{1}} c_{i j},
\end{aligned}
$$


and this contradicts (3.1)(ii).

$$
\left|A(U) \cap J_{2}\right|+p^{\prime}+\left|J_{1}\right| \geqq|U| \quad\left(U \subset \subset I, U_{2} \neq \varnothing\right) .
$$

Suppose for some $U \subset \subset I$, the inequality did not hold. Then

$$
\begin{aligned}
\sum_{i \in U} \sum_{j \in J_{2}} c_{i j} & <t\left(|U|-\left|J_{1}\right|-p^{\prime}\right), \\
t p^{\prime} & <t|U|-\sum_{i \in U} \sum_{j \in J_{2}} c_{i j}-t\left|J_{1}\right| .
\end{aligned}
$$

Because of (3.1)(ii), this implies $t p^{\prime}<t|U|-\sum_{i \in U} \sum_{j \in J} c_{i j}$ which contradicts (3.2). This completes the proof of the theorem.

Since Theorem 3.1 is a somewhat comprehensive result, it is worthwhile to state separately some special cases which are of interest.

TheOREM 3.2. Let $C=\left(c_{i j}: i \in I, j \in J\right)$ be a nonnegative integral matrix with $\sum_{j \in J} c_{i j} \leqq t(i \in I)$ and $\sum_{i \in I} c_{i j} \leqq t(j \in J)$ where $t$ is a positive integer. Let

$$
t(p-1)+1 \leqq \sum_{i \in I}\left(t-\sum_{j \in J} c_{i j}\right) \leqq t p^{\prime}
$$

where $p$ is a nonnegative integer, $p^{\prime}$ is a nonnegative integer or $\infty$ with $p \leqq p^{\prime}$ where if $p>0$, then $p^{\prime}<\infty$. Then there exists a subpermutation matrix $P$ with the properties: (i) $P \leqq C$, (ii) $P$ has $a 1$ in each row and column of $C$ which sums to $t$, (iii) $p \leqq$ (row defect of $P) \leqq p^{\prime}$.

This follows from Theorem 3.1 by setting $q=0$ and $q^{\prime}=\infty$.

TheOREM 3.3. Let $C=\left(c_{i j}: i \in I, j \in J\right)$ be a nonnegative integral matrix with $\sum_{j \in J} c_{i j} \leqq t(i \in I)$ and $\sum_{i \in I} c_{i j} \leqq t(j \in J)$ where $t$ is a positive integer. Let

$$
t(p-1)+1 \leqq \sum_{i \in I}\left(t-\sum_{j \in J} c_{i j}\right) \leqq t p, \quad t(q-1)+1 \leqq \sum_{j \in J}\left(t-\sum_{i \in I} c_{i j}\right) \leqq t q
$$

where $p$ and $q$ are nonnegative integers. Then there exists a subpermutation matrix $P$ such that (i) $P \leqq C$, (ii) $P$ has $a 1$ in each row and column of $C$ which sums to $t$, (iii) (row defect of $P)=p,($ column defect of $P)=q$.

This is the special case of Theorem 3.1 obtained when $p^{\prime}=p, q^{\prime}=q$.

We mention one final special case before deriving some representation theorems for integral matrices.

TheOREM 3.4. Let $C=\left(c_{i j}: i \in I, j \in J\right)$ be a nonnegative integral matrix with $\sum_{j \in J} c_{i j} \leqq t(i \in I)$ and $\sum_{i \in I} c_{i j} \leqq t(j \in J)$ where $t$ is a positive integer. Then there exists a permutation matrix $P$ with $P \leqq C$ such that $P$ has $a 1$ in each row and column of $C$ which sums to $t$.

This is the special case of Theorem 3.1 obtained by setting $p=q=0, p^{\prime}=q^{\prime}=\infty$. 
From Theorem 3.1 and its special cases follow a number of results on representing integral matrices as sums of subpermutation matrices. All of these results, including Theorems 3.1 to 3.4 , reduce to known results when the matrix is taken to be finite. We shall collect our results in one theorem.

THEOREM 3.5. Let $C=\left(c_{i j}: i \in I, j \in J\right)$ be a nonnegative integral matrix with $\sum_{j \in J} c_{i j} \leqq t(i \in I)$ and $\sum_{i \in I} c_{i j} \leqq t(j \in J)$. Let $p^{\prime}$ and $q^{\prime}$ be nonnegative integers.

(I) If $\sum_{i \in I}\left(t-\sum_{j \in J} c_{i j}\right) \leqq t p^{\prime}, \sum_{j \in J}\left(t-\sum_{i \in I} c_{i j}\right) \leqq t q^{\prime}$, then $C=P_{1}+\cdots+P_{t}$ where $P_{1}, \ldots, P_{t}$ are subpermutation matrices with row defect at most $p^{\prime}$ and column defect at most $q^{\prime}$.

(II) If $\sum_{i \in I}\left(t-\sum_{j \in J} c_{i j}\right)=t p^{\prime}, \sum_{j \in J}\left(t-\sum_{i \in I} c_{i j}\right)=t q^{\prime}$, then $C=P_{1}+\cdots+P_{t}$ where $P_{1}, \ldots, P_{t}$ are subpermutation matrices with row defect equal to $p^{\prime}$ and column defect equal to $q^{\prime}$.

(III) If $\sum_{i \in I}\left(t-\sum_{j \in J} c_{i j}\right) \leqq t p^{\prime}$, then $C=P_{1}+\cdots+P_{t}$, where $P_{1}, \ldots, P_{t}$ are subpermutation matrices with row defect at most $p^{\prime}$.

(IV) If $\sum_{i \in I}\left(t-\sum_{j \in J} c_{i j}\right)=t p^{\prime}$, then $C=P_{1}+\cdots+P_{t}$, where $P_{1}, \ldots, P_{t}$ are subpermutation matrices with row defect equal to $p^{\prime}$.

(V) With no additional assumptions, $C=P_{1}+\cdots+P_{t}$ where $P_{1}, \ldots, P_{t}$ are subpermutation matrices.

Proof. We shall only indicate the derivations of (I) and (II) from Theorem 3.3. Statements (III) and (IV) will follow in a similar way from Theorem 3.2, while (V) follows immediately by induction on $t$ from Theorem 3.4 .

Choose nonnegative integers $p_{1} \leqq p^{\prime}$ and $q_{1} \leqq q^{\prime}$ so that

$$
\begin{aligned}
& t\left(p_{1}-1\right)+1 \leqq \sum_{i \in I}\left(t-\sum_{j \in J} c_{i j}\right) \leqq t p_{1}, \\
& t\left(q_{1}-1\right)+1 \leqq \sum_{j \in J}\left(t-\sum_{i \in I} c_{i j}\right) \leqq t q_{1} .
\end{aligned}
$$

By Theorem 3.3, there exists a subpermutation matrix $P=\left(p_{i j}: i \in I, j \in J\right)$ with row defect equal to $p_{1}$ and column defect equal to $q_{1}$ such that the matrix $C^{\prime}=C-P$ $=\left(c_{i j}^{\prime}: i \in I, j \in J\right)$ is a nonnegative integral matrix satisfying

$$
\sum_{j \in J} c_{i j}^{\prime} \leqq t-1 \quad(i \in I), \quad \sum_{i \in I} c_{i j}^{\prime} \leqq t-1 \quad(j \in J) .
$$

We calculate that

$$
\begin{aligned}
\sum_{i \in I}\left(t-1-\sum_{j \in J} c_{i j}^{\prime}\right) & =\sum_{i \in I}\left(t-\sum_{j \in J}\left(p_{i j}+c_{i j}^{\prime}\right)\right)-p_{1} \\
& =\sum_{i \in I}\left(t-\sum_{j \in J} c_{i j}\right)-p_{1} \\
& \leqq t p_{1}-p_{1}=(t-1) p_{1} .
\end{aligned}
$$


Likewise, $\sum_{j \in J}\left(t-1-\sum_{i \in I} c_{i j}^{\prime}\right) \leqq(t-1) q_{1}$. Hence by induction, $C^{\prime}$ is the sum of $t-1$ subpermutation matrices with row defect at most $p_{1}$ and column defect at most $q_{1}$. Since $C=P+C^{\prime}$ and $p_{1} \leqq p^{\prime}, q_{1} \leqq q^{\prime}$, (I) follows. To obtain (II) we simply use Theorem 3.3, to conclude we may take $P$ to have row defect equal to $p^{\prime}$ and column defect equal to $q^{\prime}$.

COROLlaRY 1. Let $C=\left(c_{i j}: i \in I, j \in J\right)$ be a nonnegative integral matrix whose row and column sums are bounded. The minimum value of $k$ for which there exist subpermutation matrices $P_{1}, \ldots, P_{k}$ such that $C=P_{1}+\cdots+P_{k}$ equals the maximum of the row and column sums of $C$.

The corollary is an immediate consequence of part (V) of Theorem 3.5. For finite matrices, part (V), and thus the above corollary, is well known and is generally attributed to König [13] (for an exposition of this result see [15]). Likewise (IV) for finite matrices is known and is due to Dulmage and Mendelsohn [3] ((II) is not more general than (IV) for finite matrices). This can be formulated as follows.

Let $C=\left(c_{i j}: 1 \leqq i \leqq m, 1 \leqq j \leqq n\right)$ be a finite nonnegative integral matrix. Suppose $\sum_{j=1}^{n} c_{i j} \leqq t(1 \leqq i \leqq m)$ and $\sum_{i=1}^{m} c_{i j} \leqq t(1 \leqq j \leqq n)$ and

$$
\sum_{i=1}^{m} \sum_{j=1}^{n} c_{i j}=p t
$$

where $p$ is a nonnegative integer. Then $C=P_{1}+\cdots+P_{t}$ where $P_{1}, \ldots, P_{t}$ are subpermutation matrices of rank $p$.

This follows from (IV) because (3.13) is equivalent to

$$
\sum_{i=1}^{m}\left(t-\sum_{j=1}^{n} c_{i j}\right)=(m-p) t .
$$

Hence $C=P_{1}+\cdots+P_{t}$ where $P_{1}, \ldots, P_{t}$ are subpermutation matrices with row defect equal to $(m-p)$ (that is, rank $p$ ). Actually Dulmage and Mendelsohn prove a somewhat more comprehensive result. Namely, if (3.13) is replaced by

$$
\sum_{i=1}^{m} \sum_{j=1}^{n} c_{i j}=p t+q \quad(0 \leqq q<p)
$$

then $C=P_{1}+\cdots+P_{t}+Q$ when $P_{1}, \ldots, P_{t}$ are subpermutation matrices of rank $p$ and $Q$ is a subpermutation matrix of rank $q$. (This is entirely equivalent to Theorem 1 of [3] which, because of the introduction of unnecessary parameters, is difficult to take in.) We can generalize this as follows.

TheOREM 3.6. Let $C=\left(c_{i j}: i \in I, j \in J\right)$ be a nonnegative integral matrix with $\sum_{j \in J} c_{i j} \leqq t(i \in I)$ and $\sum_{i \in I} c_{i j} \leqq t(j \in J)$. Suppose that

$$
\sum_{i \in I}\left(t-\sum_{j \in J} c_{i j}\right)=t p-q
$$


where $p$ is a nonnegative integer and $q$ is a nonnegative integer such that $q+p \leqq|I|$ (this is automatically satisfied if $I$ is an infinite set). Then

$$
C=P_{1}+\cdots+P_{t}+Q
$$

where $P_{1}, \ldots, P_{t}$ are subpermutation matrices with row defect $p$ and $Q$ is a subpermutation matrix of rank $q$.

Proof. Suppose we can show that there is a subpermutation matrix of rank $q$ such that $Q \leqq C$. The matrix $C^{\prime}=C-Q$ is then a nonnegative integral matrix with row and column sums at most $t$, and it is easily verified that

$$
\sum_{i \in I}\left(t-\sum_{j \in J} c_{i j}^{\prime}\right)=t p .
$$

By (IV) of Theorem 3.3, $C^{\prime}$ is the sum of $t$ subpermutation matrices with row defect equal to $p$, and the theorem will have been proved.

If we consider again the family $\mathfrak{X}(I)=\left(A_{i}: i \in \mathcal{I}\right)$ where $A_{i}=\left\{j \in J: c_{i j} \neq 0\right\}$, then we need to show that $\mathfrak{A}$ has a partial transversal $T$ with $|T|=q$ and (as a consequence of more general results given in [1] and [16]), this is equivalent to $|A(K)|+|I \backslash K| \geqq q(K \subseteq I)$. Suppose for some $K \subseteq I,|A(K)|+|I| K \mid<q$. Then

$$
\begin{aligned}
& \sum_{i \in K} \sum_{j \in J} c_{i j}<t(q-|I \backslash K|), \\
& t|I \backslash K|+ \sum_{i \in K} \sum_{j \in J} c_{i j}<t q, \\
& \sum_{i \in I} \sum_{j \in J} c_{i j}<t q .
\end{aligned}
$$

If $I$ is an infinite set, this is impossible. If $I$ is a finite set, then

$$
\begin{aligned}
\sum_{i \in I} \sum_{j \in J} c_{i j} & <t(|I|-p), \\
t p & <t|I|-\sum_{i \in I} \sum_{j \in J} c_{i j} .
\end{aligned}
$$

But this contradicts (3.14) (since $q \geqq 0$ ), and the theorem is proved.

The theorem of Dulmage and Mendelsohn results when $I$ and $J$ are finite sets, for then (3.14) becomes

$$
\sum_{i \in I} \sum_{j \in J} c_{i j}=t(|I|-p)+q
$$

where $0 \leqq q \leqq|I|-p$.

4. Common partial transversals. We shall be concerned in this section with certain packing and covering problems associated with the common partial transversals of two families $\mathfrak{A}$ and $\mathfrak{B}$ of subsets of a set $E$. In particular, we shall give a solution to the packing problem of order $t$ associated with the collection of common partial transversals of $\mathfrak{A}$ and $\mathfrak{B}$ having defect at most $p$ in $\mathfrak{A}$ and defect at most $q$ 
in $\mathfrak{B}$. For finite families this will reduce to the theorem proved by Fulkerson [8]. We shall also solve the covering problem of order $t$ associated with the collection of all common partial transversals of $\mathfrak{A}$ and $\mathfrak{B}$. Our proofs will be based on Theorem 2.2 and the representation properties of integral matrices given in Theorem 3.5.

We shall make use of the following notation. If $\mathfrak{A}=\left(A_{i}: i \in I\right)$ is a family of subsets of a set $E$ and $t$ is a positive integer, then $\mathfrak{X}^{(t)}$ denotes the family obtained from $\mathfrak{A}$ by repeating each of the $A$ 's $t$ times. Formally, if $Z_{t}=\{1,2, \ldots, t\}$, then $\mathfrak{U}^{(t)}=\left(A_{i, k}^{(t)}:(i, k) \in I \times Z_{t}\right)$ where $A_{i, k}^{(t)}=A_{i}\left(i \in I, k \in Z_{t}\right)$.

Before establishing our main packing result about common partial transversals, we first establish a 'linking principle' which is of considerable interest by itself. No finiteness conditions are necessary.

THEOREM 4.1. Let $\mathfrak{2}(I)=\left(A_{i}: i \in I\right)$ and $\mathfrak{B}(J)=\left(B_{j}: j \in J\right)$ be two families of subsets of a set $E$. Let $t$ be a positive integer, and let $p$ and $q$ be nonnegative integers or $\infty$. Let $T \subseteq E$. Then the following three statements, (4.1), (4.2), and (4.3), are equivalent.

(4.1) $T$ is a common partial transversal of the families $\mathfrak{A}^{(t)}$ and $\mathfrak{B}^{(t)}$ with $d_{\mathfrak{Q}}(t)(T) \leqq p t$ and $d_{\mathfrak{B}^{(t)}}(T) \leqq q t$.

(4.2) (i) There is a family $\left(T_{i}^{\prime}: 1 \leqq i \leqq t\right)$ of pairwise disjoint sets with $T=\bigcup_{i=1}^{t} T_{i}^{\prime}$ such that $T_{i}^{\prime}$ is a partial transversal of $\mathfrak{A}$ with $d_{\mathfrak{U}}\left(T_{i}\right) \leqq p(1 \leqq i \leqq t)$.

(ii) There is a family $\left(T_{i}^{\prime \prime}: 1 \leqq i \leqq t\right)$ of pairwise disjoint sets with $T=\bigcup_{i=1}^{t} T_{i}^{\prime \prime}$ such that $T_{i}^{\prime \prime}$ is a partial transversal of $\mathfrak{B}$ with $d_{\mathfrak{B}}\left(T_{i}^{\prime \prime}\right) \leqq q(1 \leqq i \leqq t)$.

(4.3) There is a family $\left(T_{i}: 1 \leqq i \leqq t\right)$ of pairwise disjoint sets with $T=\bigcup_{i=1}^{t} T_{i}$ such that $T_{i}$ is a common partial transversal of $\mathfrak{A}$ and $\mathfrak{B}$ with $d_{\mathfrak{A}}\left(T_{\mathfrak{i}}\right) \leqq p$ and $d_{\mathfrak{B}}\left(T_{\mathfrak{i}}\right) \leqq q$ $(1 \leqq i \leqq t)$.

Proof. It is clear that (4.3) implies both (4.1) and (4.2). The power of the theorem lies in the other implications. It is also clear that (4.2) implies (4.1). Thus it is only necessary to establish that (4.1) implies (4.3).

Suppose (4.1) is valid. Then there exist injections $\sigma: T \rightarrow I \times Z_{t}$ and $\theta: T \rightarrow J \times Z_{t}$ such that $x \in A_{\sigma(x)}^{(t)}(x \in T), x \in B_{\theta(x)}^{(t)}(x \in T),\left|\left\{I \times Z_{t}\right\}\right| \sigma(T) \mid \leqq p t$, and $\left|\left\{J \times Z_{t}\right\}\right| \theta(T) \mid$ $\leqq q t$. We construct a nonnegative integral matrix $C=\left(c_{i j}: i \in I, j \in J\right)$ by defining

$$
c_{i j}=\left|\left\{x: x \in T, \sigma(x) \in\{i\} \times Z_{t}, \theta(x) \in\{j\} \times Z_{t}\right\}\right| \quad(i \in I, j \in J) .
$$

The matrix $C$ then has the following properties:

(i) $\sum_{j \in J} c_{i j} \leqq t(i \in I), \sum_{i \in I} c_{i j} \leqq t(j \in J)$.

(ii) $\sum_{i \in I}\left(t-\sum_{j \in J} c_{i j}\right) \leqq p t, \sum_{j \in J}\left(t-\sum_{i \in I} c_{i j}\right) \leqq q t$.

By (I), (III), or (V) of Theorem 3.5 there exist subpermutation matrices $P_{1}, \ldots, P_{t}$ with row defects at most $p$ and column defects at most $q$ such that $C=P_{1}+\cdots+P_{t}$. But this means that there exist pairwise disjoint sets $T_{1}, \ldots, T_{t}$ whose union is $T$ and injections $\sigma_{i}: T_{i} \rightarrow I, \theta_{i}: T_{i} \rightarrow J$ such that $x \in A_{\sigma_{i}(x)}, y \in B_{\theta_{i}(x)}\left(x \in T_{i}, 1 \leqq i \leqq t\right)$ where $|I| \sigma_{i}(T) \mid \leqq p$ and $\left|J \backslash \theta_{i}(T)\right| \leqq q(1 \leqq i \leqq t)$. That is, $T_{i}$ is a common partial 
transversal of $\mathfrak{A}$ and $\mathfrak{B}$ with $d_{\mathfrak{A}}\left(T_{i}\right) \leqq p, d_{\mathfrak{B}}\left(T_{i}\right) \leqq q(1 \leqq i \leqq t)$. This completes the proof of the theorem.

By choosing $p$ and $q$ to be 0 or $\infty$, some special cases which are of interest result. Continuing with the terminology of Theorem 4.1 we record two of these (but only the part which says that (4.2) and (4.3) are equivalent).

THEOREM 4.2. The following two statements are equivalent.

(4.4) There is a family $\left(T_{i}: 1 \leqq i \leqq t\right)$ of pairwise disjoint sets with $T=\bigcup_{i=1}^{t} T_{i}$ such that $T_{i}$ is a common transversal of $\mathfrak{U}$ and $\mathfrak{B}(1 \leqq i \leqq t)$.

(4.5) (i) There is a family $\left(T_{i}^{\prime}: 1 \leqq i \leqq t\right)$ of pairwise disjoint sets with $T=\bigcup_{i=1}^{t} T_{i}^{\prime}$ such that $T_{i}^{\prime}$ is a transversal of $\mathfrak{A}(1 \leqq i \leqq t)$.

(ii) There is a family $\left(T_{i}^{\prime \prime}: 1 \leqq i \leqq t\right)$ of pairwise disjoint sets with $T=\bigcup_{i=1}^{t} T_{i}^{\prime \prime}$ such that $T_{i}^{\prime \prime}$ is a transversal of $\mathfrak{B}(1 \leqq i \leqq t)$.

This is the special case $p=q=0$ of Theorem 4.1 .

THEOREM 4.3. The following two statements are equivalent.

(4.6) There is a family $\left(T_{i}: 1 \leqq i \leqq t\right)$ with $T=\bigcup_{i=1}^{t} T_{i}$ such that $T_{i}$ is a common partial transversal of $\mathfrak{A}$ and $\mathfrak{B}(1 \leqq i \leqq t)$.

(4.7) (i) There is a family $\left(T_{i}^{\prime}: 1 \leqq i \leqq t\right)$ with $T=\bigcup_{i=1}^{t} T_{i}^{\prime}$ such that $T_{i}^{\prime}$ is a partial transversal of $\mathfrak{A}(1 \leqq i \leqq t)$.

(ii) There is a family $\left(T_{i}^{\prime \prime}: 1 \leqq i \leqq t\right)$ with $T=\bigcup_{i=1}^{t} T_{i}^{\prime \prime}$ such that $T_{i}^{\prime \prime}$ is a partial transversal of $\mathfrak{B}(1 \leqq i \leqq t)$.

This follows from Theorem 4.1 by choosing $p=q=\infty$ and from the fact that a subset of a partial transversal (resp. common partial transversal) is also a partial transversal (resp. common partial transversal).

We now solve the packing problem for common transversals.

TheOREM 4.4. Let $\mathfrak{A}(I)=\left(A_{i}: i \in I\right)$ and $\mathfrak{B}(J)=\left(B_{j}: j \in J\right)$ be two families of subsets of a set $E$, and suppose each element of $E$ is a member of only finitely many $A$ 's and B's. Let $M \subseteq E$, let $p$ and $q$ be nonnegative integers or $\infty$, and let $t$ be $a$ nonnegative integer. If $p<\infty$, assume $A_{i}$ is a finite set $(i \in I)$; if $q<\infty$, assume $B_{j}$ is a finite set $(j \in J)$. Then there exists a family $\left(T_{i}: 1 \leqq i \leqq t\right)$ of pairwise disjoint sets such that (i) $M \subseteq \bigcup_{i=1}^{t} T_{i}$, (ii) $T_{i}$ is a common partial transversal of $\mathfrak{A}$ and $\mathfrak{B}$ $(1 \leqq i \leqq t)$, (iii) $d_{\mathfrak{V l}}\left(T_{i}\right) \leqq p, d_{\mathfrak{B}}\left(T_{i}\right) \leqq q(1 \leqq i \leqq t)$ if and only if

$$
\begin{array}{cc}
|A(K) \cap B(J \backslash L)|+|\{A(K) \cup B(J \backslash L)\} \cap N|+t p \geqq t(|K|-|L|)+|N| \\
(K \subset \subset I, L \subset \subset J, N \subset \subset M) \\
|A(I \backslash K) \cap B(L)|+|\{A(I \backslash K) \cup B(L)\} \cap N|+t q \geqq t(|L|-|K|)+|N| \\
(K \subset \subset I, L \subset \subset J, N \subset \subset M) \\
|A(I \backslash K) \cap N|+t|K| \geqq|N| & (K \subset \subset I, N \subset \subset M) \\
|B(J \backslash L) \cap N|+t|L| \geqq|N| & (L \subset \subset J, N \subset \subset M) .
\end{array}
$$


Proof. According to Theorem 4.1 the existence of a family $\left(T_{i}: 1 \leqq i \leqq t\right)$ of pairwise disjoint sets with properties (i), (ii), (iii) is equivalent to the existence of a set $T \subseteq E$ with $M \subseteq T$ such that $T$ is a common partial transversal of $\mathfrak{A}^{(t)}$ and $\mathfrak{B}^{(t)}$ with $d_{\mathfrak{Q}^{(t)}}(T) \leqq p t, d_{\mathfrak{B}^{(t)}}(T) \leqq q t$. Conditions for the existence of such a $T$ are given in Theorem 2.2. We assert that the conditions corresponding to (2.5), (2.6), (2.7), and (2.8) are, respectively, (4.7), (4.8), (4.9), (4.10). We show only the first of these, the other verifications being quite similar. Corresponding to (2.5) we have the cordition

$$
\begin{aligned}
\left|A^{(t)}(P) \cap B^{(t)}\left(\left\{J \times Z_{t}\right\} \mid Q\right)\right|+\left|\left\{A^{(t)}(P) \cup B^{(t)}\left(\left\{J \times Z_{t}\right\} \mid Q\right)\right\} \cap N\right|+t p \\
\\
\geqq|P|-|Q|+N \quad\left(P \subset \subset I \times Z_{t}, Q \subset \subset J \times Z_{t}, N \subset \subset M\right) .
\end{aligned}
$$

Let $N \subset \subset M$ be fixed. Choose $K \subset \subset I$ minimal and $L \subset \subset J$ maximal with respect to the properties $P \subseteq K \times Z_{t},\left\{J \times Z_{t}\right\} \mid Q \subseteq\{J \backslash L\} \times Z_{t}$. Then the above inequality can be written as

$$
|A(K) \cap B(J \backslash L)|+|\{A(K) \cup B(J \backslash L)\} \cap N|+t p \geqq|P|-|Q|+|N| .
$$

Now for fixed $K$ and $L$, the left side of this inequality is independent of $K$ and $L$ while the right side is largest when $P=K \times Z_{t},\left\{J \times Z_{t}\right\} \backslash Q=\{J \backslash L\} \times Z_{t}$. If $P$ and $Q$ are chosen thus, then $|P|=t|K|$ and $|Q|=t|L|$, so that the conditions of (4.11) are equivalent to those of (4.7). This completes the proof.

Continuing with the assumptions of Theorem 4.4 we record some special cases which are of interest.

COROLlaRY 1. There exists a family $\left(T_{i}: 1 \leqq i \leqq t\right)$ of pairwise disjoint sets such that $T_{i}$ is a common partial transversal of $\mathfrak{A}$ and $\mathfrak{B}$ with $d_{\mathfrak{A}}\left(T_{i}\right) \leqq p, d_{\mathfrak{B}}\left(T_{i}\right) \leqq q(1 \leqq i \leqq t)$ if and only if

$$
\begin{array}{ll}
|A(K) \cap B(J \backslash L)|+t p \geqq t(|K|-|L|) & (K \subset \subset I, L \subset \subset J), \\
|A(I \backslash K) \cap B(L)|+t q \geqq t(|L|-|K|) & (K \subset \subset I, L \subset \subset J) .
\end{array}
$$

This is the special case of Theorem 4.4 obtained by taking $M=\varnothing$.

COROLlaRY 2. There exists a family $\left(T_{i}: 1 \leqq i \leqq t\right)$ of pairwise disjoint sets such that $T_{i}$ is a common transversal of $\mathfrak{A}$ and $\mathfrak{B}(1 \leqq i \leqq t)$ if and only if

$$
\begin{aligned}
|A(K) \cap B(J \backslash L)| \geqq t(|K|-|L|) & & (K \subset \subset I, L \subset \subset J), \\
|A(I \backslash K) \cap B(L)| \geqq t(|L|-|K|) & & (K \subset \subset I, L \subset \subset J) .
\end{aligned}
$$

This is the special case of Corollary 1 obtained by choosing $p=q=0$.

COROllary 3. There exists a family $\left(T_{i}: 1 \leqq i \leqq t\right)$ of pairwise disjoint sets such that $T_{i}$ is a transversal of $\mathfrak{A}$ and a partial transversal of $\mathfrak{B}(1 \leqq i \leqq t)$ if and only if

$$
|A(K) \cap B(J \backslash L)| \geqq t(|K|-|L|) \quad(K \subset \subset I, L \subset \subset J) .
$$


This is the special case of Theorem 4.4 arrived at by taking $p=0, q=\infty$. While the $A$ 's must be finite sets, the $B$ 's need not be.

There are other interesting special cases which we leave to the reader to formulate, except that we show how to obtain Fulkerson's theorem [8] from Theorem 4.4.

COROLlary 4. If the families $\mathfrak{A}$ and $\mathfrak{B}$ are finite families and $k$ is a nonnegative integer, then there exists a family $\left(T_{i}: 1 \leqq i \leqq t\right)$ of pairwise disjoint sets with $\left|T_{i}\right|=k$ such that $T_{i}$ is a common partial transversal of $\mathfrak{A}$ and $\mathfrak{B}(1 \leqq i \leqq t)$ if and only if

$$
|A(K) \cap B(L)| \geqq t(k-|I \backslash K|-|J \backslash L|) \quad(K \subseteq I, L \subseteq J) .
$$

To obtain this from Theorem 4.4, take $M=\varnothing, p=|I|-k, q=\infty$.

We now turn to the covering problem of order $t$ associated with the common partial transversals of two families of sets.

THEOREM 4.5. Let $\mathfrak{A}(I)=\left(A_{i}: i \in I\right)$ and $\mathfrak{B}(J)=\left(B_{j}: j \in J\right)$ be two families of subsets of a set $E$. Assume that each element of $E$ is a member of only finitely many $A$ 's and B's. Let $t$ be a positive integer, and let $p, q$ be nonnegative integers or $\infty$. If $p<\infty$ assume that $A_{i}$ is a finite set $(i \in I)$; if $q<\infty$, assume that $B_{j}$ is a finite set $(j \in J)$.

Then there exists a family $\left(T_{i}: 1 \leqq i \leqq t\right)$ of pairwise disjoint sets such that $E=\bigcup_{i=1}^{t} T_{i}$ where $T_{i}$ is a common partial transversal of $\mathfrak{A}$ and $\mathfrak{B}$ with $d_{\mathfrak{A}}\left(T_{i}\right) \leqq p$, $d_{\mathfrak{B}}\left(T_{i}\right) \leqq q(1 \leqq i \leqq t)$ if and only if the following conditions are satisfied.

(4.12) $|A(K)|+t p \geqq t|K|(K \subset \subset I)$,

(4.13) $|A(I \backslash K) \cap F|+t|K| \geqq|F|(K \subset \subset I, F \subset \subset E)$,

(4.14) $|B(L)|+t q \geqq t|L|(L \subset \subset J)$,

(4.15) $|B(J \backslash L)|+t|L| \geqq|F|(L \subset \subset J, F \subset \subset E)$.

Indeed (4.12) and (4.13) are equivalent to the existence of a family $\left(T_{i}^{\prime}: 1 \leqq i \leqq t\right)$ of pairwise disjoint sets such that $E=\bigcup_{i=1}^{t} T_{i}^{\prime}$ where $T_{i}^{\prime}$ is a partial transversal of I with $d_{\mathfrak{A}}\left(T_{i}^{\prime}\right) \leqq p(1 \leqq i \leqq t)$. A similar statement applies to (4.14) and (4.15).

Proof. By Theorem 4.1, a family $\left(T_{i}: 1 \leqq i \leqq t\right)$ having the desired properties exists if and only if $E$ is a partial transversal of $\mathfrak{A}^{(t)}$ with defect at most $p t$ and a partial transversal of $\mathfrak{B}^{(t)}$ with defect at most $q t$. It is sufficient to show that the first of these is equivalent to the conditions (4.12) and (4.13). By Corollary 1 to Theorem 2.2, $E$ is a partial transversal of $\mathfrak{2}^{(t)}$ with defect at most $p t$ if and only if

(4.16) $\left|A^{(t)}(P)\right|+p t \geqq|P|(P \subset \subset I)$,

(4.17) $\left|A^{(t)}(I \backslash P) \cap F\right|+|P| \geqq|F|(P \subset \subset I, F \subset \subset E)$.

We assert that (4.12) is equivalent to (4.16) and (4.13) is equivalent to (4.17). Since the proof of these involves calculations similar to the ones done in the proof of Theorem 4.1, we omit them. The theorem is proved.

We now record some special cases of Theorem 4.5.

THEOREM 4.6. Let $\mathfrak{A}(I)=\left(A_{i}: i \in I\right)$ and $\mathfrak{B}(J)=\left(B_{j}: j \in J\right)$ be two families of finite subsets of a set $E$ such that each element of $E$ is a member of only finitely many 
$A$ 's and B's. Then there is a family $\left(T_{i}: 1 \leqq i \leqq t\right)$ of pairwise disjoint sets where $E=\bigcup_{i=1}^{t} T_{i}$ such that $T_{i}$ is a common transversal of $\mathfrak{A}$ and $\mathfrak{B}(1 \leqq i \leqq t)$ if and only if

(4.18) $|A(K)| \geqq t|K|(K \subset \subset I)$,

(4.19) $|A(K) \cap F|+t|K| \geqq|F|(K \subset \subset I, F \subset \subset E)$,

(4.20) $|B(L)| \geqq t|L|(L \subset \subset J)$,

(4.21) $|B(L) \cap F|+t|L| \geqq|F|(L \subset \subset J, F \subset \subset E)$.

This is the special case of Theorem 4.5 obtained by choosing $p=q=0$. Observe that this solves the 'simultaneous' packing and covering problem for the common transversals of $\mathfrak{A}$ and $\mathfrak{B}$. The solution of the covering problem for common partial transversals is the content of the next theorem.

THEOREM 4.7. Let $\mathfrak{A}(I)=\left(A_{i}: i \in I\right)$ and $\mathfrak{B}(J)=\left(B_{j}: j \in J\right)$ be two families of subsets of a set $E$. Assume that each element of $E$ is a member of only finitely many A's and B's. Then there exists a family $\left(T_{i}: 1 \leqq i \leqq t\right)$ of sets such that $E=\bigcup_{i=1}^{t} T_{i}$ where $T_{\mathfrak{i}}$ is a common partial transversal of $\mathfrak{A}$ and $\mathfrak{B}$ if and only if

(4.22) $|A(I \backslash K) \cap F|+t|K| \geqq|F|(K \subset \subset I, F \subset \subset E)$,

(4.23) $|B(J \backslash L) \cap F|+t|L| \geqq|F|(L \subset \subset J, F \subset \subset E)$.

This theorem follows from Theorem 4.5 by choosing $p=q=\infty$. If desired, the family $\left(T_{i}: 1 \leqq i \leqq t\right)$ may be chosen to consist of pairwise disjoint sets. This is so, because a subset of a common partial transversal is also a common partial transversal.

Theorems 4.5, 4.6 and 4.7 also contain theorems about the partial transversals of one family of sets. For instance, if $\mathfrak{A}=\left(A_{i}: i \in I\right)$ is a family of finite subsets of a set $E$ such that each element of $E$ is a member of only finitely many $A$ 's, then (4.18) and (4.19) are necessary and sufficient for there to exist a family $\left(T_{i}: 1 \leqq i \leqq t\right)$ of pairwise disjoint sets such that $\bigcup_{i=1}^{t} T_{i}=T$ where $T_{i}$ is a transversal of $\mathfrak{A}$. Observe that (4.22) is necessary and sufficient for $E=\bigcup_{i=1}^{t} T_{i}^{\prime}$ where $T_{i}^{\prime}$ is a partial transversal of $\mathfrak{A}$; a similar statement holds for (4.23).

5. Concluding remarks. It is possible to solve also the packing problem of order $t$ associated with the collection of common partial transversals of cardinality $p<\infty$ of two arbitrary (not necessarily finite) families of two sets. This will be a generalization in a different direction of Fulkerson's result (Corollary 4 of Theorem 4.4 in this paper). In order to do this we need an additional result on common partial transversals.

Lemma 5.1. Let $\mathfrak{A}(I)=\left(A_{i}: i \in I\right)$ and $\mathfrak{B}(J)=\left(B_{j}: j \in J\right)$ be families of subsets of a set $E$. Let $k$ be a nonnegative integer. Then $\mathfrak{A}$ and $\mathfrak{B}$ have a common partial transversal $T$ with $|T|=k$ if and only if

$$
|A(I \backslash P) \cap B(J \backslash Q)|+|P|+|Q| \geqq k \quad(P \subset \subset I, Q \subset \subset J) .
$$

This lemma can be derived from more general known results (Theorem 3.8 of [2], or [16]). 
Lemma 5.2. Let $C=\left(c_{i j}: i \in I, j \in J\right)$ be a nonnegative integral matrix. Let $p$ and $t$ be positive integers and suppose

$$
\sum_{j \in J} c_{i j} \leqq t \quad(i \in I), \quad \sum_{i \in I} c_{i j} \leqq t \quad(j \in J), \quad \sum_{i \in I} \sum_{j \in J} p_{i j}=p t .
$$

Then $C=P_{1}+\cdots+P_{t}$ where $P_{1}, \ldots, P_{t}$ are subpermutation matrices of rank $p$.

This follows from the corresponding result when $I$ and $J$ are finite sets, since the conditions imply that $\left\{i \in I: c_{i j}>0\right.$ for some $\left.j \in J\right\}$ and $\left\{j \in J: c_{i j}>0\right.$ for some $\left.i \in I\right\}$ are finite sets.

TheOREM 5.2. Let $\mathfrak{A}(I)=\left(A_{i}: i \in I\right)$ and $\mathfrak{B}(J)=\left(B_{j}: j \in J\right)$ be families of subsets of a set $E$. Let $p$ and $t$ be positive integers. Then there exists a family $\left(T_{i}: 1 \leqq i \leqq t\right)$ of pairwise disjoint sets where $T_{i}$ is a common partial transversal of $\mathfrak{A}$ and $\mathfrak{B}$ with $\left|T_{i}\right|=p(1 \leqq i \leqq t)$ if and only if

$$
|A(I \backslash K) \cap B(J \backslash L)|+t|K|+t|L| \geqq p t \quad(K \subset \subset I, L \subset \subset J) .
$$

Proof. We only sketch the proof, since the procedure is the same as the one used before. Using Lemma 5.2, one shows that the required family $\left(T_{i}: 1 \leqq i \leqq t\right)$ exists if and only if $\mathfrak{Y}^{(t)}$ and $\mathfrak{B}^{(t)}$ have a common partial transversal with cardinality pt. Applying Lemma 5.1 to $\mathfrak{A}^{(t)}$ and $\mathfrak{B}^{(t)}$ with $k=p t$, one obtains the conditions (5.1).

It is possible to improve Theorem 5.2 by requiring that $\bigcup_{i=1}^{t} T_{i}$ contain a prescribed set $M$. In order to do this one has to improve Lemma 5.1 in the corresponding way. This also can be done by using the more general results in [1] or [16]. We leave this to the interested reader.

\section{REFERENCES}

1. R. A. Brualdi, A very general theorem on systems of distinct representatives, Trans. Amer. Math. Soc. 140 (1969), 149-160.

2. - A general theorem concerning common transversals, Proc. Conference Combinatorial Mathematics and its Applications (Oxford, 1969), Academic Press, New York, 1971.

3. A. L. Dulmage and N. S. Mendelsohn, Some graphical properties of matrices with nonnegative entries, Aequationes Math. 2 (1969), 150-162.

4. J. Edmonds, Minimum partition of a matroid into independent subsets, J. Res. Nat. Bur. Standards Sect. B 69B (1965), 67-72. MR 32 \#7441.

5. J. Edmonds and D. R. Fulkerson, Transversals and matroid partition, J. Res. Nat. Bur. Standards Sect. B 69B (1965), 147-153. MR 32 \#5531.

6. J. Folkman and D. R. Fulkerson, Flows in infinite graphs, J. Combinatorial Theory 8 (1970), 30-44.

7. L. R. Ford, Jr. and D. R. Fulkerson, Network flow and systems of representatives, Canad. J. Math. 10 (1958), 78-84. MR 20 \#4502.

8. D. R. Fulkerson, Disjoint common partial transversals of $t$ wo families of sets (to appear).

9. P. Hall, On representatives of subsets, J. London Math. Soc. 10 (1935), 26-30.

10. M. Hall, Jr., Distinct representatives of subsets, Bull. Amer. Math. Soc. 54 (1948), 922-926. MR 10, 238. 
11. P. J. Higgins, Disjoint transversals of subsets, Canad. J. Math. 11 (1959), 280-285. MR 21 \#3341.

12. A. J. Hoffman and H. W. Kuhn, On systems of distinct representatives. Linear inequalities and related systems, Ann. of Math. Studies, no. 38, Princeton Univ. Press, Princeton, N. J., 1956, pp. 199-206. MR 18, 416.

13. D. König, Theorie der endlichen und unendlichen Graphen. Kombinatorische Topologie der Streckenkomplexe, Chelsea, New York, 1950. MR 12, 195.

14. L. Mirsky, Transversals of subsets, Quart. J. Math. Oxford Ser. (2) 17 (1966), 58-60. MR 34 \#2477.

15. - - Pure and applied combinatorics, Bull. Inst. Math. Appl. 5 (1969), 2-4.

16. H. Perfect, $A$ generalization of Rado's theorem on independent transversals, Proc. Cambridge Philos. Soc. 66 (1969), 513-515. MR 39 \#5382.

UNIVERSITY OF SHEFFIELD,

SHEFFIELD, ENGLAND

UNIVERSITY OF WISCONSIN,

MADISON, WisCONSIN 53706 